\title{
SOME RESULTS IN THE LINEAR DYNAMICAL THEORY OF ANISOTROPIC ELASTIC SOLIDS*
}

\author{
$\mathrm{BY}$
}

\author{
LEWIS T. WHEELER
}

University of Houston

Introduction. This paper aims at generalizations to anisotropic homogeneous elastic solids of some of the results contained in a recent comprehensive study [1] of various topics in the linearized dynamical theory of homogeneous and isotropic elastic media. All of the theorems proved here encompass unbounded domains. Specifically, we establish the prolonged quiescence of the far elastodynamic field in an initially quiescent anisotropic body. In addition, we prove a uniqueness theorem and a reciprocal identity which are applicable to infinite anisotropic solids, in the absence of artificial regularity requirements at infinity. With these objectives in mind, we first deduce an appropriate elastodynamic analogue of Zaremba's [2] generalized energy identity for the scalar wave equation.

1. Notation and preliminaries. The notation used in this paper is, except for minor modifications, the same as that employed in [1]. Ordinary lower-case letters stand for scalars, while lower-case boldface Latin letters denote vectors and lower-case Grcek letters in boldface designate higher-order tensors. If $\mathbf{x}$ is the position vector of a point in the euclidean three-space $E$, we write

$$
B_{\delta}(\mathbf{x})=\{\mathbf{z}|\mathbf{z} \in E,| \mathbf{z}-\mathbf{x} \mid<\delta\} \quad(\delta>0)
$$

for a spherical ball of radius $\delta$ centered at $\mathbf{x}$. In particular, we let $B_{\delta}$ stand for $B_{\delta}(0)$. The letter $R$ at present will always denote a regular region in $E$ : thus $R$ is open and there exists $\delta_{0}>0$ such that for every $\delta>\delta_{0}$ the set $R \cap B_{\delta}$ is connected and has as its boundary a finite number of nonintersecting "closed regular surfaces", the latter term being used in the sense of Kellogg [3, p. 112]. If $\partial R$ is the boundary ${ }^{1}$ of $R$, we call $\partial^{*} R$ the set of all points in $\partial R$ at which its normal is defined. The symbol $T^{\infty}$ is reserved for the entire real line and $T$ denotes an arbitrary (open, closed, or half-open) interval of $T^{\infty}$. In addition, we use the notation

$$
T^{-}=(-\infty, 0], T^{+}=[0, \infty) .
$$

Standard indicial notation, with the usual summation and differentiation conventions, is employed in connection with cartesian tensor fields of any order. Also, we write $\varphi \cdot \psi$ for the fully contracted outer product $\varphi_{i j} \ldots_{k} \psi_{i} \ldots_{k}$ of two tensors $\varphi, \psi$ of the same order

${ }^{*}$ Received January 10, 1969; revised version received April 28, 1969. This investigation was carried out under Contract Nonr-220(58) of the California Institute of Technology with the Office of Naval Research in Washington, D. C. The author is grateful to Eli Sternberg for encouraging the research described here and for many valuable discussions during the preparation of the manuscript.

${ }^{1}$ Throughout this paper, we use the conventional notations for the boundary, the interior, and the closure of a set, as well as for the cartesian product of two sets. 
and $\nabla \mathbf{v}$ for the tensor with the components $v_{i, i}$, if $\mathbf{v}$ is a differentiable vector field. Finally, to facilitate concise smoothness hypotheses, we employ the function classes $\mathfrak{e}(P)$ and $\mathfrak{e}^{\alpha}(P)$. The class $\mathfrak{e}(P)$ consists of all tensor-valued functions of any order that are defined and continuous on a subset $P$ of euclidean $n$-space; for $\alpha$ a positive integer, $\mathfrak{C}^{\alpha}(P)$ consists of all functions in $\mathfrak{C}(P)$ whose partial derivatives of order up to and including $\alpha$ exist on $\stackrel{\odot}{P}$ and there coincide with functions belonging to $\mathcal{C}(P)$.

As for preliminaries from the linear dynamical theory of elasticity, we require the notion of an

Elastodynamic state. If $\mathbf{u}$ and $\mathbf{d}$ are, respectively, a vector-valued and a second-order tensor-valued function defined on $\bar{R} \times T$, we call the ordered pair $[\mathbf{u}, \boldsymbol{\sigma}]$ an elastodynamic state on $\bar{R} \times T$ corresponding to the body-force field $\mathbf{f}$, the mass density $\rho$, and to the elasticity tensor $\gamma$, and write

$$
[\mathbf{u}, \mathbf{d}] \in \varepsilon(\mathbf{f}, \boldsymbol{\rho}, \boldsymbol{\gamma} ; \bar{R} \times T),
$$

provided:

(a) $\mathbf{u} \in \mathbb{e}^{2}(R \times T) \cap \mathfrak{e}^{1}(\bar{R} \times T), \boldsymbol{d} \in \mathbb{e}(\bar{R} \times T), \mathbf{f} \in \mathfrak{e}(\bar{R} \times T)$, $\rho$ is a positive constant, and $\gamma$ is a constant fourth-order tensor such that

$$
\begin{gathered}
\gamma_{i j k l}=\gamma_{i i k l}=\gamma_{k l i j}, \\
\gamma_{i j k l} \varphi_{i j} \varphi_{k l} \geq 0 \text { for every second-order tensor } \varphi ;
\end{gathered}
$$

(b) $\mathfrak{u}, \boldsymbol{o}, \mathbf{f}, \rho$, and $\boldsymbol{\gamma}$ on $R \times \stackrel{\circ}{\mathrm{T}}$ satisfy the equations

$$
\begin{aligned}
& \sigma_{i j, j}+f_{i}=\rho \ddot{u}_{i}, \\
& \sigma_{i j}=\gamma_{i j k l} u_{k, l} .
\end{aligned}
$$

If, in particular,

$$
T=T^{\infty}, \quad \mathbf{u}=\mathbf{0} \text { on } \bar{R} \times T^{-},
$$

we say that $[\mathbf{u}, \mathbf{d}]$ is an elastodynamic state with a quiescent past and write

$$
[\mathbf{u}, \boldsymbol{\sigma}] \in \mathcal{E}_{0}(\mathbf{f}, \rho, \boldsymbol{\gamma} ; \bar{R}) .
$$

Eqs. (1.5) represent the stress equations of motion and Eqs. (1.6) the stress-displacement relations of the linear theory. Note that (1.3), (1.6), together with the assumed continuity of $\boldsymbol{d}$ on $\bar{R} \times T$, imply the symmetry of $\boldsymbol{d}$ on $\bar{R} \times T$. Condition (1.4) assures that the quadratic function $e$ defined by

$$
e(\varphi)=\frac{1}{2} \gamma_{i j k l} \varphi_{i j} \varphi_{k l} \text { for every second-order tensor } \varphi,
$$

is positive semi-definite. If $\mathbf{u}$ is the displacement field of an elastodynamic state, then $e(\nabla \mathfrak{u})$ evidently represents the strain-energy density associated with this state. Finally, if $\boldsymbol{\sigma}$ is the stress field of an elastodynamic state on $\bar{R} \times T$ and $\mathbf{n}$ is the unit outward normal of $\partial^{*} R$, we call the vector field s defined by

$$
s_{i}=\sigma_{i j} n_{i} \text { on } \partial^{*} R \times T,
$$

the tractions of $\boldsymbol{d}$ acting on $\partial R$.

2The superposed dots indicate partial time-differentiation. 
2. Prolonged quiescence of the far elastodynamic field. Uniqueness and reciprocal theorems. In preparation for the theorems that constitute our main objective, we require the following auxiliary result.

Lemma 1 (Generalized energy identity). Suppose:

(a) $[\mathbf{u}, \boldsymbol{\sigma}] \in \varepsilon_{0}(\mathbf{f}, \rho, \boldsymbol{\gamma} ; \bar{R})$;

(b) $\tau \in \mathfrak{C}^{1}(\bar{R})$ is a given function such that the set

$$
\mathbf{x} \mid \mathbf{x} \in \bar{R}, \tau(\mathbf{x})>0\}
$$

is bounded. Let $\mathbf{\psi}$ be the second-order tensor-valued function defined by

$$
\psi_{i j}(\mathbf{x})=\dot{u}_{i}(\mathbf{x}, \tau(\mathbf{x})) \tau_{, i}(\mathbf{x}) \text { for every } \mathbf{x} \in R .
$$

Then,

$$
\begin{aligned}
\int_{\partial R} \int_{0}^{\tau(\mathbf{x})} \dot{\mathfrak{u}}(\mathbf{x}, t) \cdot \mathbf{s}(\mathbf{x}, t) d t d A+\int_{R} \int_{0}^{\tau(\mathbf{x})} \dot{\mathfrak{u}}(\mathbf{x}, t) \cdot \mathbf{f}(\mathbf{x}, t) d t d V \\
=\int_{R}\left[\frac{\rho}{2} \dot{\mathbf{u}}^{2}(\mathbf{x}, \tau(\mathbf{x}))-e(\psi(\mathbf{x}))+e(\nabla \mathbf{u}(\mathbf{x}, \tau(\mathbf{x}))+\psi(\mathbf{x}))\right] d V,
\end{aligned}
$$

where $\mathbf{s}$ are the tractions of $\mathbf{d}$ acting on $\partial R$ and $e$ is given by (1.9).

Proof. For convenience define vector fields $\mathbf{p}$ and $\mathbf{v}$ through

$$
p_{i}=\dot{u}_{i} \sigma_{i i} \quad \text { on } \quad \bar{R} \times T^{\infty}, \quad v_{i}(\mathrm{x})=\int_{0}^{\tau(\mathrm{x})} p_{i}(\mathrm{x}, t) d t \quad \text { for every } \quad \mathrm{x} \in \bar{R} .
$$

The degree of smoothness of $\mathbf{u}, \boldsymbol{\delta}, \tau$ implied by hypotheses (a), (b) ensures that

$$
\mathrm{v} \in \mathfrak{e}^{1}(R) \cap \mathfrak{e}(\bar{R}) .
$$

Next, note that (2.3), (1.5) furnish

$$
\begin{aligned}
\nabla \cdot \mathrm{v}(\mathrm{x})=\int_{0}^{\tau(\mathrm{x})}[\nabla \dot{\mathbf{u}}(\mathrm{x}, t) \cdot \boldsymbol{\mathrm { o }}(\mathrm{x}, t)+ & \left.\frac{\rho}{2} \frac{\partial}{\partial t} \dot{\mathfrak{u}}^{2}(\mathrm{x}, t)-\dot{\mathfrak{u}}(\mathrm{x}, t) \cdot \mathrm{f}(\mathrm{x}, t)\right] d t \\
& +\mathrm{p}(\mathrm{x}, \tau(\mathrm{x})) \cdot \nabla \tau(\mathrm{x}) \quad \text { for all } \quad \mathrm{x} \in R,
\end{aligned}
$$

while (1.6), (1.3), (1.9) yield

$$
\nabla \dot{\mathbf{u}}(\mathbf{x}, t) \cdot \mathbf{o}(\mathbf{x}, t)=\frac{\partial}{\partial t} e(\nabla \mathbf{u}(\mathbf{x}, t)) \quad \text { for all } \quad(\mathbf{x}, t) \in R \times T^{\infty} .
$$

Substitute from (2.6) into (2.5) and use (1.7) to arrive at

$$
\nabla \cdot \mathrm{v}(\mathbf{x})=\mathbf{p}(\mathbf{x}, \tau(\mathbf{x})) \cdot \nabla \tau(\mathbf{x})+e(\nabla \mathbf{u}(\mathbf{x}, \tau(\mathbf{x})))-\int_{0}^{\tau(\mathbf{x})} \dot{\mathbf{u}}(\mathbf{x}, t) \cdot \mathbf{f}(\mathbf{x}, t) d t+\frac{\rho}{2} \dot{\mathbf{u}}^{2}(\mathbf{x}, \tau(\mathbf{x})),
$$

which, after a brief computation involving (1.6), (2.3), (1.9), (2.1) and the symmetry relations (1.3), gives rise to

$$
\begin{aligned}
\nabla \cdot \mathrm{v}(\mathrm{x})=e(\nabla \mathfrak{u}(\mathrm{x}, \tau(\mathrm{x}))+\mathrm{\psi}(\mathrm{x})) & -e(\mathrm{u}(\mathrm{x}))+(\rho / 2) \dot{\mathrm{u}}^{2}(\mathrm{x}, \tau(\mathrm{x})) \\
& -\int_{0}^{\tau(\mathrm{x})} \dot{\mathrm{u}}(\mathrm{x}, t) \cdot \mathrm{f}(\mathrm{x}, t) d t \quad \text { for every } \quad \mathrm{x} \in R .
\end{aligned}
$$


Observe that $\mathbf{v}$ has bounded support by virtue of (2.3), hypothesis (b), and (1.7). Moreover, hypothesis (b) and (1.7), because of (1.9), (2.1), imply that each term in the right-hand member of (2.7) is the value at $\mathbf{x}$ of a function with bounded support. Thus, the individual terms in the right-hand member of (2.7), and hence $\nabla \cdot v$, are properly integrable on $R$, since (1.9), (2.1) and the assumed regularity of $\mathbf{u}, \mathbf{f}, \tau$ imply that each of these terms represents the value at $\mathbf{x}$ of a function in $\mathcal{e}(\bar{R})$. The preceding remarks concerning $\mathbf{v}$ and $\nabla \cdot \mathbf{v}$, in conjunction with (2.4), entitle one to apply the divergence theorem ${ }^{3}$ to $\mathrm{v}$ on $R$. Accordingly, (2.7) leads to

$$
\begin{aligned}
\int_{\partial R} \mathbf{v}(\mathbf{x}) \cdot \mathbf{n}(\mathbf{x}) d A=\int_{R}\left[\frac{\rho}{2} \dot{\mathbf{u}}^{2}(\mathbf{x}, \tau(\mathbf{x}))-e(\psi(\mathbf{\psi}(\mathbf{x}))+e(\nabla \mathbf{u}(\mathbf{x}, \tau(\mathbf{x}))+\mathbf{\psi}(\mathbf{x}))] d V\right. \\
\\
\quad-\int_{R} \int_{0}^{\tau(\mathbf{x})} \dot{\mathbf{u}}(\mathbf{x}, t) \cdot \mathbf{f}(\mathbf{x}, t) d t d V,
\end{aligned}
$$

where $\mathbf{n}$ is the unit outward normal of $\partial^{*} R$. The desired result (2.2) now follows at once with the aid of (2.3) and (1.10). This completes the proof.

If $R$ is bounded, we may in particular choose $\tau=t$ on $\bar{R}$, where $t$ is any given positive number; for this choice (2.2) passes over into the classical energy identity

$$
\begin{aligned}
\int_{\partial R} \int_{0}^{t} \dot{\mathbf{u}}(\mathbf{x}, \lambda) \cdot \mathbf{s}(\mathbf{x}, \lambda) d \lambda d A+\int_{R} \int_{0}^{t} \dot{\mathbf{u}}(\mathbf{x}, \lambda) \cdot \mathbf{f}(\mathbf{x}, \lambda) d \lambda d V \\
=\int_{R}\left[\frac{\rho}{2} \dot{\mathbf{u}}^{2}(\mathbf{x}, t)+e(\nabla \mathbf{u}(\mathbf{x}, t))\right] d V .
\end{aligned}
$$

The preceding lemma reduces to Lemma 2.1 of [1] in the special case of isotropy, for which

$$
\gamma_{i i k l}=\mu\left(\delta_{i k} \delta_{i l}+\delta_{i l} \delta_{i k}+\frac{2 \nu}{1-2 \nu} \delta_{i j} \delta_{k l}\right),
$$

where $\mu$ and $\nu$ respectively denote the shear modulus and Poisson's ratio, while $\delta_{i j}$ is the Kronecker delta. We now use Lemma 1 to establish

Theorem 1 (Sufficient conditions for the prolonged quiescence of the FAR ELASTODYNAMIC FIELD). Let $R$ be unbounded and suppose

(a) $[\mathbf{u}, \boldsymbol{b}] \in \varepsilon_{0}(\mathbf{f}, \rho, \boldsymbol{\gamma} ; \bar{R})$;

(b) for every $t>0$, there is a bounded set $\Lambda(t) \subset \bar{R}$ such that

$$
\mathbf{f}=\mathbf{0} \text { on }(\bar{R}-\Lambda(t)) \times[0, t],
$$

and, if $\partial R$ extends to infinity,

$$
\dot{\mathrm{u}} \cdot \mathbf{s}=0 \text { on }\left(\partial^{*} R-\Lambda(t)\right) \times[0, t],
$$

where $\mathbf{s}$ is given by (1.10).

Then, for every $t>0$, there exists a bounded set $\Omega(t) \subset \bar{R}$, depending only on $\Lambda(t), \rho$, and $\boldsymbol{\gamma}$, such that

$$
\mathbf{u}=\mathbf{\sigma}=\mathbf{0} \text { on }(\bar{R}-\Omega(t)) \times[0, t] .
$$

${ }^{3}$ See Theorem 1.1 of [1] for the required version of the divergence theorem, which is a trivial modification of the strongest form stated and proved by Kellogg in [3, p. 11.9]. 
Proof. It is clear from (1.9) that

$$
e(\varphi) \leq \frac{\rho}{4} c^{2} \varphi \cdot \varphi \quad \text { for every second-order tensor } \varphi,
$$

provided

$$
c=\sqrt{2(1+81|\gamma| / \rho)}, \quad|\gamma|=\sqrt{\gamma \cdot \gamma} .
$$

With $c$ so determined, fix $t>0$, and choose $\delta>0$ such that

$$
\begin{aligned}
& \partial R \cup \Lambda(t) \subset \bar{B}_{\delta} \quad \text { if } \quad \partial R \text { is bounded, } \\
& \Lambda(t) \subset \bar{B}_{\delta} \quad \text { if } \quad \partial R \text { is unbounded. }
\end{aligned}
$$

Now consider the set

$$
\Omega(t)=\bar{R} \cap \bar{B}_{\delta+c t} .
$$

Since $\Omega(t)$ is closed, one shows readily ${ }^{4}$ that $\bar{R}-\Omega(t)$ is contained in the closure of $R-\Omega(t)$. In view of the continuity of $\mathbf{u}, \boldsymbol{\sigma}$ on $\bar{R} \times T^{\infty}$, and because (1.6) holds on $R \times T^{\infty}$, it suffices to show that

$$
\mathbf{u}=\mathbf{0} \text { on }(R-\Omega(t)) \times[0, t]
$$

in order to establish (2.10). To this end, choose

$$
(\mathrm{z}, \lambda) \in(R-\Omega(t)) \times(0, t]
$$

and hold $(z, \lambda)$ fixed. Define

$$
\tau(\mathrm{x})=\lambda-\frac{1}{c}|\mathrm{x}-\mathrm{z}| \quad \text { for every } \mathrm{x} \in \bar{R} .
$$

If $R_{z}$ and $\bar{R}_{z}$ stand for the sets obtained from $R$ and $\bar{R}$ by deletion of the point $\mathrm{z}$, then evidently

$$
\begin{gathered}
\tau \in \mathbb{e}^{1}\left(\bar{R}_{z}\right) \cap \mathfrak{e}(\bar{R}), \quad|\nabla \tau(\mathbf{x})|=\frac{1}{c} \quad \text { for all } \quad \mathrm{x} \in R_{\mathbf{z}}, \\
\{\mathbf{x} \mid \mathbf{x} \in \bar{R}, \tau(\mathbf{x})>0\}=\bar{R} \cap B_{c \lambda}(\mathbf{z}) .
\end{gathered}
$$

It follows from (2.14), (2.16) that $\bar{B}_{\delta}$ does not intersect $B_{c \lambda}(\mathrm{z})$, so that (2.13), (2.19) imply

$$
\begin{aligned}
& \{\mathbf{x} \mid \mathbf{x} \in \bar{R}, \tau(\mathbf{x})>0\} \subset \bar{R}-\Lambda(t) \cup \partial R \text { if } \partial R \text { is bounded, } \\
& \{\mathbf{x} \mid \mathbf{x} \in \bar{R}, \tau(\mathbf{x})>0\} \subset \bar{R}-\Lambda(t) \quad \text { if } \partial R \text { is unbounded. }
\end{aligned}
$$

Next, appeal to (2.16), (2.17) to see that

$$
\tau(\mathbf{x}) \leq t \text { for every } \mathbf{x} \in \bar{R} .
$$

Hypothesis (b), (2.20), (2.21), and the fact that $\mathbf{u}$ vanishes on $\bar{R} \times T^{-}$according to hypothesis (a), furnish 


$$
\begin{array}{ll}
\int_{0}^{r(\mathrm{x})} \dot{\mathrm{u}}(\mathrm{x}, \eta) \cdot \mathrm{f}(\mathrm{x}, \eta) d \eta=0 & \text { for every } \quad \mathrm{x} \in \bar{R} \\
\int_{0}^{\tau(\mathrm{x})} \dot{\mathrm{u}}(\mathrm{x}, \eta) \cdot \mathrm{s}(\mathrm{x}, \eta) d \eta=0 & \text { for every } \mathrm{x} \in \partial^{*} R .
\end{array}
$$

At this stage, consider the one-parameter family of regular regions $R(\xi)$ given by

$$
R(\xi)=R-\bar{B}_{\xi}(\mathrm{z}) \quad\left(0<\xi<\xi_{0}\right)
$$

with $\xi_{0}>0$ such that $\bar{B}_{\xi_{0}}(\mathbf{z}) \subset R$. Observe that the present elastodynamic state [u, $\left.\mathbf{0}\right]$ and the function $\tau$ defined by (2.17) satisfy the hypotheses of Lemma 1 if $\bar{R}$ in this lemma is replaced by $\bar{R}(\xi)\left(0<\xi<\xi_{0}\right)$. Invoking Lemma 1 , taking account of (2.22), and subsequently passing to the limit as $\xi \rightarrow 0$, one easily obtains

$$
\int_{R}\left[\frac{\rho}{2} \dot{\mathbf{u}}^{2}(\mathbf{x}, \tau(\mathbf{x}))-e(\psi(\mathbf{x}))+e(\nabla \mathbf{u}(\mathbf{x}, \tau(\mathbf{x}))+\mathbf{\psi}(\mathbf{x}))\right] d V=0
$$

where $\psi$ has the components

$$
\psi_{i j}(\mathbf{x})=\dot{u}_{i}(\mathbf{x}, \tau(\mathbf{x})) \tau_{, i}(\mathbf{x}) \text { for all } \mathbf{x} \in R_{\mathbf{z}},
$$

while $e$ is defined by (1.9).

In view of hypothesis (a), $e$ is positive semidefinite. Hence (2.11), (2.24), (2.18) imply that

$$
\begin{aligned}
\frac{\rho}{2} \dot{\mathbf{u}}^{2}(\mathbf{x}, \tau(\mathbf{x}))-e(\psi(\mathbf{x})) & \geq \frac{\rho}{2} \dot{\mathbf{u}}^{2}(\mathbf{x}, \tau(\mathbf{x}))-\frac{\rho}{4} c^{2} \psi(\mathbf{x}) \cdot \psi(\mathbf{x}) \\
& =\frac{\rho}{2} \dot{\mathbf{u}}^{2}(\mathbf{x}, \tau(\mathbf{x}))-\frac{\rho}{4} c^{2} \dot{\mathbf{u}}^{2}(\mathbf{x}, \tau(\mathbf{x}))[\nabla \tau(\mathbf{x})]^{2} \\
& =\frac{\rho}{4} \dot{\mathbf{u}}^{2}(\mathbf{x}, \tau(\mathbf{x})) \geq 0 \quad \text { for every } \quad \mathbf{x} \in R_{\mathbf{z}} .
\end{aligned}
$$

Therefore, the integrand in (2.23) is nonnegative on $R_{z}$. Also, this integrand is continuous on $R_{z}$, as a consequence of (1.9), (2.24), the first of (2.18), and the smoothness of $\mathbf{u}$ implied by hypothesis (a). These observations, together with (2.23), yield

$$
\frac{\rho}{2} \dot{\mathbf{u}}^{2}(\mathbf{x}, \tau(\mathbf{x}))-e(\boldsymbol{\psi}(\mathbf{x}))+e(\nabla \mathbf{u}(\mathbf{x}, \tau(\mathbf{x}))+\boldsymbol{\psi}(\mathbf{x}))=0 \quad \text { for every } \quad \mathbf{x} \in R_{\mathbf{z}},
$$

which, in conjunction with (2.25), implies

$$
\dot{\mathbf{u}}(\mathbf{x}, \tau(\mathbf{x}))=\mathbf{0} \text { for every } \mathbf{x} \in R_{\mathbf{z}} \text {. }
$$

But $\dot{\mathfrak{u}}$ is continuous on $\bar{R} \times T^{\infty}$, and $\tau$ is continuous on $R$. Accordingly, (2.27), (2.17) lead to

$$
\dot{\mathrm{u}}(\mathrm{z}, \lambda)=\dot{\mathrm{u}}(\mathrm{z}, \tau(\mathrm{z}))=\mathbf{0},
$$

and thus, since $(\mathbf{z}, \lambda)$ was chosen arbitrarily in $(R-\Omega(t)) \times(0, t]$,

$$
\dot{\mathbf{u}}=\mathbf{0} \text { on }(R-\Omega(t)) \times(0, t] .
$$

The assertion (2.15), and consequently (2.10), now follow at once from (1.7) and the 
assumed regularity of $\mathfrak{u}$. Finally, note from (2.12), (2.13), (2.14) that $\Omega(t)$ depends exclusively on $\Lambda(t), \rho$, and $\gamma$. The proof is now complete.

Theorem 1 contains as a special case Lemma 2.2 of [1], which is confined to the isotropic medium characterized by (2.9). We are now ready to state and prove

Theorem 2 (Generalized uniqueness theorem). Suppose

(a) $\left[\mathbf{u}^{\prime}, \mathbf{o}^{\prime}\right] \in \mathcal{\varepsilon}\left(\mathbf{f}, \rho, \boldsymbol{\gamma} ; \bar{R} \times T^{+}\right),\left[\mathbf{u}^{\prime \prime}, \boldsymbol{o}^{\prime \prime}\right] \in \mathcal{E}\left(\mathbf{f}, \rho, \boldsymbol{\gamma} ; \bar{R} \times T^{+}\right)$;

(b) $\mathbf{u}^{\prime}(\mathbf{x}, 0)=\mathfrak{u}^{\prime \prime}(\mathbf{x}, 0), \dot{\mathfrak{u}}^{\prime}(\mathbf{x}, 0+)=\dot{\mathfrak{u}}^{\prime \prime}(\mathbf{x}, 0+)$ for every $\mathbf{x} \in R$;

(c) $\left(\dot{\mathbf{u}}^{\prime}-\dot{\mathbf{u}}^{\prime \prime}\right) \cdot\left(\mathbf{s}^{\prime}-\mathbf{s}^{\prime \prime}\right)=0$ on $\partial^{*} R \times[0, \infty)$, where $\mathbf{s}^{\prime}$ and $\mathbf{s}^{\prime \prime}$ are the tractions of $\mathbf{b}^{\prime}$ and $\mathbf{b}^{\prime \prime}$ acting on $\partial R$.

Then,

$$
\mathbf{u}^{\prime}=\mathbf{u}^{\prime \prime}, \mathbf{o}^{\prime}=\mathbf{o}^{\prime \prime} \text { on } \bar{R} \times T^{+} .
$$

Proof. Define $\mathfrak{u}, \boldsymbol{d}$ on $\bar{R} \times T^{\infty}$ through

$$
\mathbf{u}=\mathbf{u}^{\prime}-\mathbf{u}^{\prime \prime}, \quad \boldsymbol{\sigma}=\boldsymbol{\sigma}^{\prime}-\boldsymbol{\sigma}^{\prime \prime} \text { on } \bar{R} \times T^{+}, \quad \mathbf{u}=\boldsymbol{\sigma}=\mathbf{0} \text { on } \bar{R} \times \stackrel{\circ}{T}^{-} .
$$

Hypotheses (a), (b) allow one to conclude without difficulty that

$$
[\mathfrak{u}, \boldsymbol{\sigma}] \in \mathcal{E}_{0}(\mathbf{0}, \rho, \boldsymbol{\gamma} ; \bar{R}) .
$$

If $\mathbf{s}$ denotes the tractions of $\boldsymbol{d}$ acting on $\partial R$, then (2.29), (2.30), (1.10), and hypothesis (c) imply that

$$
\dot{\mathrm{u}} \cdot \mathbf{s}=0 \text { on } \dot{o}^{*} R \times T^{*} .
$$

Choose $t>0$ and consider $t$ as fixed for the remainder of the argument. For a bounded $R$ one draws from (2.30), (2.31), and the energy identity (2.8) that

$$
\int_{R}\left[\frac{\rho}{2} \dot{\mathbf{u}}^{2}(\mathbf{x}, t)+e(\nabla \mathbf{u}(\mathbf{x}, t))\right] d V=0
$$

with $e$ given by (1.9). Suppose next that $R$ is unbounded. In this event (2.30), (2.31), and Theorem 1 guarantee the existence of a bounded set $\Omega(t) \subset \bar{R}$ for which (2.10) holds. Let

$$
R(\delta)=R \cap B_{\delta}(\delta>0),
$$

and choose $\delta$ large enough to ensure that $R(\delta)$ is a regular region ${ }^{6}$ and

$$
\Omega(t) \subset B_{\delta} .
$$

Since $R(\delta)$ is a bounded set contained entirely in $R,(2.30),(2.8)$ yield

$$
\int_{\partial R(\delta)} \int_{0}^{t} \dot{\mathbf{u}}(\mathbf{x}, \lambda) \cdot \mathbf{s}(\mathbf{x}, \lambda) d \lambda d A=\int_{R(\delta)}\left[\frac{\rho}{2} \dot{\mathbf{u}}^{2}(\mathbf{x}, t)+e(\nabla \mathbf{u}(\mathbf{x}, t))\right] d V,
$$

provided $\mathbf{s}$ here denotes the tractions of $\boldsymbol{d}$ acting on $\partial R(\delta)$. It is clear from (2.31), (2.33), (2.34), and (2.10) that the integrand in the left-hand member of (2.35) vanishes for $(\mathbf{x}, \lambda) \in \partial R(\delta) \times[0, t]$, whereas the integrand in the right-hand member vanishes for all $\mathbf{x} \in R-R(\delta)$. Hence (2.32) remains valid also if $R$ is unbounded.

The argument may now be completed along the lines of the classical uniqueness

${ }^{6}$ Recall the definition of $R$ in Section 1. 
proof. Indeed, since $e$ is positive semidefinite, and because of the continuity of the integrand in (2.32) for every $\mathbf{x} \in R$, one has

$$
\dot{\mathbf{u}}(\mathbf{x}, t)=\mathbf{0} \text { for all } \mathbf{x} \in R .
$$

Thus, $t$ having been chosen arbitrarily in $(0, \infty)$,

$$
\dot{\mathrm{u}}=\mathbf{0} \text { on } R \times(0, \infty) .
$$

This fact, in view of (2.29), (2.30), confirms the desired conclusion (2.28).

The preceding theorem is an extension to anisotropic solids of Theorem 2.1 in [1]. Theorem 2 is also broader than the latter theorem because it covers the general mixed problem, in addition to the first and the second fundamental problem of dynamic elasticity. We note that the foregoing proof of Theorem 2, which relies on the prolonged quiescence of the far field established in Theorem 1, is considerably more economical than the procedure used in arriving at Theorem 2.1 of [1]. The present method of proof is an adaptation of Neumann's [5] classical uniqueness argument to unbounded anisotropic elastic solids, in which Theorem 1 served the purpose of avoiding artificial order restrictions on the velocity and stresses at infinity.

As for the generalized reciprocal theorem, we turn first to some prerequisites from the theory of Riemann convolutions. If $P$ is a set in $E$, and if

$$
\varphi \in \mathfrak{e}\left(P \times T^{+}\right), \quad \psi \in \mathfrak{e}\left(P \times T^{+}\right),
$$

we mean by the comolution of $\varphi$ and $\psi$ the function $\varphi^{*} \psi$ defined on $P \times T^{*}$ through

$$
\begin{aligned}
{[\varphi * \psi](\mathrm{x}, t) } & =0 \quad \text { for every } \quad(\mathrm{x}, t) \in P \times T^{-} \\
& =\int_{0}^{t} \varphi(\mathrm{x}, t-\tau) \psi(\mathrm{x}, \tau) d \tau \quad \text { for every } \quad(\mathrm{x}, t) \in P \times \dot{T}^{+} .
\end{aligned}
$$

We will use without mention the commutativity and distributivity of convolutions; additional required properties of convolutions are cited in

Lemma 2. Suppose $P$ is a set in $E$ and let

$$
\theta \in \mathfrak{C}\left(P \times T^{+}\right), \quad \omega \in \mathcal{C}\left(P \times T^{+}\right) .
$$

(A) If $(\mathbf{x}, t) \in P \times(0, \infty)$ and $[\theta * \omega](\mathbf{x}, \cdot)=0$ on $[0,2 t]$, then either $\theta(\mathbf{x}, \cdot)=0$ on $[0, t]$ or $\omega(\mathbf{x}, \cdot)=0$ on $[0, t]$.

(B) If $P$ is in particular an open or closed region in $E$ and

$$
\varphi \in \mathfrak{e}^{1}\left(P \times T^{+}\right), \quad \psi \in \mathbb{e}^{1}\left(P \times T^{+}\right), \quad \chi=\varphi^{*} \psi,
$$

then

(a) $\theta * \omega \in \mathcal{C}\left(P \times T^{\omega}\right), \chi \in \mathcal{C}^{1}\left(P \times T^{+}\right)$;

(b) $\chi_{, i}=\varphi_{, i} * \psi+\varphi * \psi_{, i}$ on $\stackrel{B}{P} \times T^{+}$,

$$
\dot{\chi}(\mathbf{x}, t)=\left[\dot{\varphi}^{*} \psi\right](\mathbf{x}, t)+\varphi(\mathbf{x}, 0) \psi(\mathbf{x}, t) \text { for every }(\mathbf{x}, t) \in P \times \hat{T}^{+} ;
$$

(c) $\chi \in \mathbb{e}^{1}\left(P \times T^{\omega}\right)$, provided $\varphi(\mathbf{x}, 0)=0$ for all $\mathbf{x} \in P$.

Part (A) of this lemma is an elementary consequence of the strengthened form of Titchmarsh's theorem given in Mikusinski's book [6, p. 397]; part (B) is merely a restatement of portions of Lemmas 1.1, 1.2, and 1.3 in [1]. 
In connection with vector fields, we write $\theta * \mathrm{v}$ for the vector with the components $\theta * v_{i}$, if the latter convolutions are meaningful. The analogous interpretation is to be attached to $\theta^{*} \downarrow$, where $\psi$ is a higher-order tensor field. Finally, we adopt the notation

$$
\mathrm{v} * \mathrm{~W}=v_{i} * w_{i}, \quad \varphi * \psi=\varphi_{i j} \cdots k * \psi_{i} \cdots k
$$

provided $\varphi$ and $\psi$ have the same order and the convolutions appearing in the righthand members of (2.37) are defined.

We may now proceed to

Theorem 3 (Generalized reciprocal theorem). Let

$$
[\mathbf{u}, \boldsymbol{\jmath}] \in \mathcal{E}\left(\mathbf{f}, \rho, \boldsymbol{\gamma} ; \bar{R} \times T^{+}\right), \quad\left[\mathbf{u}^{\prime}, \mathbf{d}^{\prime}\right] \in \mathcal{\varepsilon}\left(\mathbf{f}^{\prime}, \rho, \boldsymbol{\gamma} ; \bar{R} \times T^{+}\right)
$$

and, for every $\mathbf{x} \in R$, put

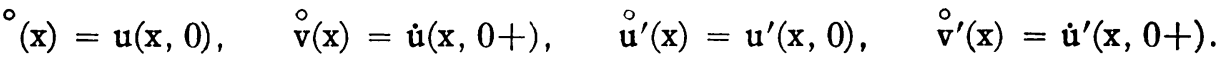

When $R$ is unbounded, suppose further:

(a) for every $t \in(0, \infty)$ there exists a bounded set $\Gamma(t) \subset \vec{R}$ such that

$$
\mathbf{f}=\mathbf{0} \text { on }(\bar{R}-\Gamma(t)) \times[0, t],
$$

and, if $\partial R$ is unbounded,

$$
\mathbf{u}(\mathbf{x}, \xi) \cdot \mathbf{s}(\mathbf{x}, \eta)=0 \quad \text { for all }(\mathbf{x}, \xi, \eta) \in\left(\partial^{*} R-\Gamma(t)\right) \times[0, t] \times[0, t],
$$

where $\mathbf{s}$ are the tractions of $\mathbf{d}$ acting on $\partial R$;

(b) each of the sets

$$
\begin{aligned}
& \Pi=\{\mathrm{x} \mid \mathrm{x} \in R, \stackrel{\circ}{\mathrm{u}}(\mathrm{x}) \neq 0\} \\
& \Xi=\{\mathrm{x} \mid \mathrm{x} \in R, \stackrel{\circ}{\mathrm{v}}(\mathrm{x}) \neq 0\}
\end{aligned}
$$

has a bounded closure.

Then, for each $t \in(0, \infty)$,

$$
\begin{aligned}
& \int_{\partial R}\left[\mathbf{s} * \mathbf{u}^{\prime}\right](\mathbf{x}, t) d A+\int_{R}\left\{\left[\mathbf{f} * \mathfrak{u}^{\prime}\right](\mathbf{x}, t)+\rho\left[\dot{\mathbf{u}}^{\prime}(\mathbf{x}, t) \cdot \stackrel{\circ}{\mathbf{u}}(\mathbf{x})+\mathbf{u}^{\prime}(\mathbf{x}, t) \cdot \stackrel{\circ}{\mathbf{v}}(\mathbf{x})\right]\right\} d V \\
& =\int_{\partial R}\left[\mathbf{s}^{\prime} * \mathbf{u}\right](\mathbf{x}, t) d A+\int_{R}\left\{\left[\mathbf{f}^{\prime} * \mathbf{u}\right](\mathbf{x}, t)+\rho\left[\dot{\mathbf{u}}(\mathbf{x}, t) \cdot \stackrel{\circ}{\mathbf{u}^{\prime}}(\mathbf{x})+\mathbf{u}(\mathbf{x}, t) \cdot \stackrel{\circ}{\mathbf{v}^{\prime}}(\mathbf{x})\right]\right\} d V,
\end{aligned}
$$

where $\mathbf{s}$ and $\mathbf{s}^{\prime}$ denote the respective tractions acting on $\partial R$.

Proof. It is convenient to show first that if $R$ is infinite, there exists, for every $t \in(0, \infty)$, a bounded set $\Omega(t) \subset \bar{R}$ such that (2.10) holds. Thus suppose $R$ is unbounded. Define

$$
\begin{array}{rlrl}
\varphi(t) & =0 & \text { for every } & t \in(-\infty, 0] \\
& =t^{3} \quad \text { for every } & t \in(0, \infty),
\end{array}
$$

and put

$$
\hat{\mathbf{u}}=\varphi * \mathbf{u}, \hat{\boldsymbol{\sigma}}=\varphi^{*} \boldsymbol{\delta} \quad \text { on } \bar{R} \times T^{\infty} .
$$


Then part (B) in Lemma 2 and the first of (2.38) enable one to confirm without difficulty that

$$
[\hat{\mathbf{u}}, \hat{\mathbf{o}}] \in \varepsilon_{0}(\hat{\mathbf{f}}, \rho, \boldsymbol{\gamma} ; \bar{R}),
$$

where

$$
\hat{\mathbf{f}}=\varphi * \mathbf{f}+\rho \varphi \stackrel{\circ}{\mathbf{V}}+\rho \dot{\varphi} \mathbf{u} \quad \text { on } \bar{R} \times T^{\infty} .
$$

Next, set

$$
\Lambda(t)=\Gamma(t) \cup \Pi \cup \Xi \text { for every } t \in(0, \infty),
$$

where $\Gamma(t)$, II, and $\Xi$ are as specified in hypotheses (a) and (b). Thus, for $t>0$,

$$
\Lambda(t) \subset \bar{R}, \Lambda(t) \text { is bounded, }
$$

and (2.46), (2.36), together with hypotheses (a), (b), imply

$$
\hat{\mathbf{f}}=\mathbf{0} \text { on }(\bar{R}-\Lambda(t)) \times[0, t] .
$$

Since $\varphi(0)=0$, (2.44) and part (B) of Lemma 2 yicld $\partial \hat{\mathbf{u}} / \partial t=\dot{\varphi} * \mathfrak{u}$. Thus if $\partial R$ is infinite and $t>0$, then (2.41), (2.47), and (2.36) give

$$
\frac{\partial \hat{\mathbf{u}}}{\partial t} \cdot \hat{\mathbf{s}}=0 \quad \text { on } \quad\left(\partial^{*} R-\Lambda(t)\right) \times[0, t],
$$

where $\hat{\mathbf{s}}$ denotes the tractions of $\hat{\boldsymbol{o}}$ acting on $\partial R$. One now infers, from (2.45), (2.48), (2.49), (2.50) and Theorem 1, the existence of a bounded set $\hat{\Omega}(t) \subset \bar{R}$ with the property

$$
\hat{\mathbf{u}}=\hat{\mathbf{o}}=\mathbf{0} \text { on }(\bar{R}-\hat{\Omega}(t)) \times[0, t] .
$$

This conclusion, because of (2.43), (2.44), and part (A) in Lemma 2, implies that (2.10) holds for every $t \in(0, \infty)$, if $\Omega(t)$ is the bounded subset of $\bar{R}$ given by $\Omega(t)=\hat{\Omega}(2 t)$, which is the desired auxiliary result. Incidentally, it is now clear from (2.36), (2.38), and hypothesis (b) that the integrals in (2.42) are proper even if $R$ is unbounded.

To establish (2.42), fix $t \in(0, \infty)$ and define a vector-valued function $\mathbf{v}$ through

$$
v_{i}(\mathbf{x})=\left[\sigma_{i i} * u_{i}^{\prime}\right](\mathbf{x}, t)-\left[\sigma_{i i}^{\prime} * u_{i}\right](\mathbf{x}, t) \quad \text { for every } \mathbf{x} \in \bar{R} .
$$

Then (2.38) and part (B) in Lemma 2 imply

$$
\begin{gathered}
\mathbf{v} \in \mathcal{C}^{1}(R) \cap \mathcal{e}(\bar{R}), \\
v_{i, i}(\mathrm{x})=\left[\sigma_{i, i, i} * u_{i}^{\prime}\right](\mathbf{x}, t)+\left[\sigma_{i, i} * u_{i, i}^{\prime}\right](\mathbf{x}, t) \\
-\left[\sigma_{j i, i}^{\prime} * u_{j}\right](\mathbf{x}, t)-\left[\sigma_{i,}^{\prime} * u_{i, i}\right](\mathbf{x}, t) \quad \text { for every } \quad \mathbf{x} \in R .
\end{gathered}
$$

By (2.53), (2.38),

$$
\begin{aligned}
\nabla \cdot \mathbf{v}(\mathbf{x})=\rho\left[\ddot{\mathbf{u}} * \mathbf{u}^{\prime}\right](\mathbf{x}, t)- & {\left[\mathbf{f} * \mathfrak{u}^{\prime}\right](\mathbf{x}, t)+\left[\mathbf{d}^{*} \nabla \mathbf{u}^{\prime}\right](\mathbf{x}, t) } \\
& -\rho[\ddot{\mathbf{u}} * \mathfrak{u}](\mathbf{x}, t)+\left[\mathbf{f}^{\prime} * \mathbf{u}\right](\mathbf{x}, t)-\left[\mathbf{d}^{\prime} * \nabla \mathfrak{u}\right](\mathbf{x}, t) .
\end{aligned}
$$

Next, appeal to (1.3) and (1.6) to see that

$$
\mathbf{d} * \nabla \mathbf{u}^{\prime}=\mathbf{d}^{\prime} * \nabla \mathbf{u} \quad \text { on } R \times(0, \infty) .
$$

Apply part (B) of Lemma 2 to $\mathbf{u} * \mathbf{u}^{\prime}$ twice in succession to arrive at

$$
\ddot{\mathbf{u}} * \mathbf{u}^{\prime}-\ddot{\mathfrak{u}}^{\prime} * \mathbf{u}=\stackrel{\circ}{\mathbf{v}^{\prime}} \cdot \mathbf{u}+\stackrel{\circ}{\mathfrak{u}^{\prime}} \cdot \dot{\mathbf{u}}-\stackrel{\circ}{\mathbf{v}} \cdot \mathbf{u}^{\prime}-\stackrel{\circ}{\mathbf{u}} \cdot \dot{\mathbf{u}}^{\prime} \quad \text { on } R \times(0, \infty),
$$




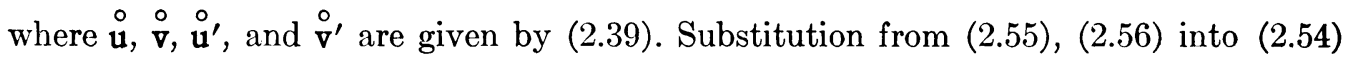
yields

$$
\begin{aligned}
\nabla \cdot \mathbf{v}(\mathbf{x})= & {\left[\mathbf{f}^{\prime} * \mathbf{u}\right](\mathbf{x}, t)+\rho\left[\stackrel{\circ}{\mathbf{v}^{\prime}}(\mathbf{x}) \cdot \mathbf{u}(\mathbf{x}, t)+\stackrel{\circ}{\mathbf{u}^{\prime}}(\mathbf{x}) \cdot \dot{\mathbf{u}}(\mathbf{x}, t)\right] } \\
& -\left[\mathbf{f} * \mathbf{u}^{\prime}\right](\mathbf{x}, t)-\rho\left[\stackrel{\circ}{\mathbf{v}}(\mathbf{x}) \cdot \mathbf{u}^{\prime}(\mathbf{x}, t)+\stackrel{\circ}{\mathbf{u}}(\mathbf{x}) \cdot \dot{\mathbf{u}}^{\prime}(\mathbf{x}, t)\right] \quad \text { for all } \quad \mathbf{x} \in R .
\end{aligned}
$$

In view of (2.51), (2.36), and the fact that if $R$ is infinite [ $\mathbf{u}, \mathbf{\sigma}]$ obeys $(2.10)$ with $\Omega(t)$ the bounded set whose existence was deduced earlier in the proof, $\mathbf{v}$ has bounded support. Equation (2.57), together with (2.38) and part (B) of Lemma 2, require $\nabla \cdot \nabla$ to coincide on $R$ with a function belonging to $\mathcal{C}(\bar{R})$. Therefore, and because of $(2.52)$, the version of the divergence theorem invoked in the proof of Lemma 1 is applicable to $\mathbf{v}$ on $R$. The identity (2.42) then follows immediately from (2.57), (2.51), and (1.10). This completes the proof.

The reciprocal identity (2.42) is due to Graffi [7], whose derivation (which is confined to bounded regions) relies on the Laplace transform. Theorem 3 is a counterpart for anisotropic bodies of Theorem 2.2 in [1], which is not only limited to the isotropic case, but also to elastodynamic states with a quiescent past. Finally, we emphasize that if the elastodynamic state $[\mathbf{u}, \boldsymbol{o}]$ of Theorem 3 is characterized as the solution to a standard boundary-initial value problem, the data enable one to decide whether or not hypotheses (a) and (b) are satisfied.

\section{REFERENCES}

[1] L. T. Wheeler and E. Sternberg, Some theorems in classical elastodynamics, Arch. Rat. Mech. Anal. 31, 51 (1968)

[2] S. Zaremba, Sopra un teorema d'unicitá relativo alla equazione delle onde sferiche, Atti Accad. Naz. Lincei (5) 24, 904 (1915)

[3] O. D. Kellogg, Foundations of potential theory, Dover, New York, 1953

[4] J. Dieudonné, Foundations of modern analysis, Academic Press, New York, 1960

[5] F. Neumann, Vorlesungen über die Theorie der Elasticität der festen Körper und des Lichtäthers, B. G. Teubner, Leipzig, 1885

[6] J. Mikusinski, Operational calculus, Pergamon, New York, 1959

[7] D. Graffi, Sul teorema di reciprocitá nella dinamica dei corpi elastici, Mem. Accad. Sci. Ist. Bologna I. Sci. Fis. (10) 4, $103(1946 / 7)$ 